\title{
The Psychology of Facial Disfigurement: Implications for Whole Face Transplantation
}

\author{
Nichola Rumsey
}

Published online: 26 June 2014

(C) Springer Science+Business Media New York 2014

\begin{abstract}
A decade has passed since the publication of the first report from the Royal College of Surgeons in England Working Party on Face Transplantation. Based on evidence available at the time, this report concluded that it was unwise to proceed with whole face transplantation (WFT). Since then, in the region of 20 WFTs have been performed. Data relating to the post-procedural psychological adjustment of a small number of patients are available and further studies have explored the psychological profiles of patients who have undergone other forms of transplantation. Understanding of the specific psychological impacts of WFT remains in its infancy, however, advances in relation to the broader psychology of disfigurement may have relevance in refining the psychological aims of WFT and the care offered to patients. A recently developed framework of psychological adjustment to disfigurement is discussed in relation to protocols for preprocedural assessment and post-operative support and intervention in WFT.
\end{abstract}

Keywords Whole face transplantation - Psychological adjustment $\cdot$ Burden of care $\cdot$ Assessment $\cdot$ Intervention

\section{Introduction}

In 2004, the Royal College of Surgeons Working Party on Face Transplantation published a report on the technical, immunological, ethical, psychological and legal aspects of

\footnotetext{
N. Rumsey $(\bowtie)$

Centre for Appearance Research, Department of Health \& Social Sciences, University of the West of England, Frenchay Campus, Bristol BS16 1QY, UK

e-mail: nichola.rumsey@uwe.ac.uk
}

facial transplantation [1]. Members appreciated the extent of psychological distress experienced by potential candidates for a whole face transplant (WFT) and were of the opinion that WFT had the potential to be a major breakthrough in relation to aesthetic outcomes; however, major concerns were expressed about the potential consequences and side effects of lifelong immunosuppression. The Report highlighted the lack of understanding in several key areas, including about the balance of risks (for example, of graft failure) and benefits, about the potential psychological impacts on the recipient, the recipients family and the donor family and about how informed consent might be achieved. In the light of these uncertainties, the Working Party concluded that it was 'unwise to proceed with human facial transplantation' in the UK at that time, recommending instead an incremental approach to the accumulation of the knowledge necessary to implement the procedure. As a member of this Working Party, it feels timely a decade later to review current knowledge about the psychological impacts of WFT and the broader field of adjustment to disfigurement.

The psychological literature available to inform the discussions of the first Working Party included studies relating to psychological responses to various types of organ transplantation and a smattering of commentaries relating to the impacts of hand transplants [1]. These studies included reports of raised levels of post-procedural anxiety relating to the short- and longer-term viability of the transplant, the aftermath of possible rejection and the potential and actual side effects of immunosuppression. The burden of personal responsibility for the success or failure of the graft, the challenges associated with the integration of the transplant into their identity and/or body image and the mismatch between pre-operative expectations of outcome and the post-procedural reality were also 
cited as being challenging for some transplant recipients. In addition, members of the Working Party debated the key role of the face in identity and in interpersonal communication, the motivation of prospective patients to seek WFT, potential mismatches in their pre-operative expectations of outcome and post-transplant reality, the burden of care (for example, adhering to lifelong immunosuppression), and the challenges of dealing with the reactions of others to the transplant. The burden for the recipient's family and the societal impacts of face transplantation were also discussed.

The Working Party reconvened following a partial transplant from a cadaver donor in France in 2005 and a second in China, the availability of medium term follow-up data for hand and forearm transplants and further articles discussing research and commentary relevant to the psychological and ethical issues raised in the 2004 report. After an examination of the new evidence, The Working Party's second report [2] concluded that the consequences of lifelong immunosuppression and its attendant complications and difficulties remained a major concern and significant reservations about the physical and psychological risks to the patient remained. The report advocated considerable caution in those planning to undertake whole face transplantation in the UK. Extensive guidance was given on the minimum requirements to be fulfilled before a transplant team should contemplate offering this procedure to patients [2]. This guidance was also designed to provide relevant information for ethical review bodies considering applications to perform WFT, in the hope that only multidisciplinary teams with appropriate experience and expertise and carefully developed protocols for the assessment of suitable patients, for pre-operative and postoperative care would be given ethical approval to undertake WFT in the UK.

Although no whole face transplantations have been carried out in the UK since the publication of the second report, a number have been carried out in other countries. Teams who have carried out WFT have reported psychological outcomes for small numbers of patients [3-5], and teams planning to offer WFT in future have published papers outlining their planned approach to pre- and posttransplant psychological assessment and support [6]. In the former, it is interesting to note that data from a small number of patients who have now undergone the procedure is prompting a change in the 'mood music' about the potential psychological benefits of WFT. The earlier rhetoric amongst some commentators as well as the media was that the procedure had the potential to provide the answer to the 'social death' of severe facial disfigurement, and to confer extensive benefits in psychological adjustment through changes to facial appearance [2, 7], however the reporting of actual outcomes reflect complex trajectories, with gains in some aspects of adjustment, alongside a lack of improvement or a worsening in others.

In the intervening period, a small number of papers have also been published outlining the post-procedural psychological profiles of patients who have undergone solid organ transplantation (SOT) [8-10]. These papers have also reinforced earlier indications that psychological adjustment post transplant is complex. Despite improvements to some aspects of quality of life, other key areas of psychological adjustment (for example, self esteem) show no improvements. Many patients experience significant difficulties in dealing with a particular set of psychological stressors, challenges and adaptive demands.

The challenge of identifying those who are sufficiently distressed by their disfigurement and/or lack of function to benefit from WFT, yet resilient to cope with the psychological challenges of WFT, and its aftermath was discussed in the second report of the Working Party [2] and has since been reiterated by those planning or undertaking WFT [3, 11]. Interestingly, members of one team at the forefront of WFT now recommend that the primary motivation for undertaking the procedure should be to improve function, rather than aesthetics [3]. In addition, there is a lack of consensus amongst prospective and current WFT teams about the psychological factors, processes and associated measures that should comprise the focus for pre- and postprocedural assessment. This lack of consensus will hamper the accumulation of knowledge in the field going forward.

In parallel to the transplant literature, research examining factors and processes contributing to psychological distress and resilience in the broader field of facial disfigurement have also advanced. These two literatures have not been linked in recent years and it now seems timely to offer clinicians working in the transplant field a synthesis of the implications of this research in the hope that this will resonate with those currently debating the most appropriate approach to the assessment, preparation and post-transplant management of patients presenting for WFT.

\section{Psychological Adjustment to Facial Disfigurement}

The negative psychological impacts of having a face that sets the affected person apart from societal norms have been cited as one of the major reason to pioneer facial transplantation during the last decade. There remains a consensus that in our appearance obsessed society, people with disfigurement are faced with significant psychological challenges, including challenges to their self perceptions (for example, self-esteem, body image), to their daily social and occupational functioning and to their psychological wellbeing (for example, as the result of negative emotions such as shame or stigma) [12•]. However, 
research has consistently highlighted a large degree of variation in the responses of those affected to disfigurement. Although many report significant difficulties and associated distress, a significant proportion adjust well, successfully managing both the curiosity and intrusive behaviour of others, and the risks to self-esteem and selfidentity to achieve a positive outlook [7]. Knowledge about the factors underpinning these individual differences in adjustment to disfigurement has advanced considerably since 2004. This knowledge has relevance to those involved in assessing the suitability of potential candidates for face transplantation and in informing the content and delivery of interventions that may be helpful as alternatives or adjuncts to WFT for those in need of psychological support.

The second Working Party Report [2] alluded to the consistent finding in the research literature that the extent of psychological distress resulting from a disfigurement is not well predicted by the type, extent or severity of the disfiguration [2, 7]. An accumulation of research clinical experience and personal accounts involving participants with a range of disfiguring conditions has further reinforced this important finding in the intervening years [13, 14]. Yet, this finding is rarely referred to in debates concerning WFT. These remain dominated by the assumption that the severity of the disfigurement is directly related to the extent of distress. Instead, research has demonstrated clearly that psychosocial factors and processes account for a much greater proportion of the variance in adjustment.

Differences in appearance result from a range of congenital anomalies (e.g. a cleft in the lip), from trauma (e.g. scarring following burns), disease (e.g. skin conditions, arthritis), or from medical or surgical interventions to treat other conditions (e.g. cancer). Until relatively recently, the majority of research in this field has been populated by condition specific studies focussing on small, often unrepresentative participant samples, the majority of whom have been recruited from hospital clinics. The findings have illustrated that the psychological impacts of disfigurement resulting from a large range of conditions are remarkably similar, however, the small sample sizes have meant that the evidence was not sufficiently strong to provide authoritative conclusions about which factors exacerbate distress and/or promote adjustment. Neither has there been a robust evidence base from which to develop interventions. Motivated to understand better the psychosocial factors contributing to adjustment to disfigurement, a UK wide collaboration of researchers and clinicians active in this field in the UK-The Appearance Research Collaboration ('ARC'*) —was convened with a view to achieving a consensus on the choice of key psychological constructs and associated measures, and at a more practical level, to provide the potential to recruit a larger, more representative participant pool. Prior to the ARC, the bulk of research has been conducted with those actively seeking treatment for their condition in hospital settings. In view of the possible bias towards those experiencing difficulty and distress in previous research, the ARC were also interested to learn more about levels of adjustment in those not actively seeking treatment at the time of study and made efforts to include these participants in their sample.

In a similar vein, models and theories developed to guide and underpin research and intervention in the field of disfigurement prior to this study had focused on psychological factors contributing to problems and difficulties. These too had been developed largely on the basis of results from patients disfigured by a particular condition (see for example, [14], in relation to cancer; [15] in relation to skin conditions). Perspectives on particular psychosocial constructs, for example, stigma [16], shame [17], social exclusion [18], and fear/avoidance [19] had helped to guide research and to provide a rationale for specific approaches to intervention, however, they had fallen short of offering a comprehensive representation of the complexity of factors involved in adjustment and to explain the predictors and concomitants of positive adjustment.

The ARC Research Program (For a Full Report, See [20•], Appendix A)

The key aims of ARC research program were to

- Determine similarities and differences in the extent and nature of psychosocial adjustment and distress in people with a range of disfiguring conditions recruited from either the community or from out-patient settings in a variety of geographical locations.

- Clarify the factors associated with distress or successful psychological adjustment in people with disfigurements.

- Generate a framework of factors contributing to adjustment designed to inform the development of a comprehensive package of screening, support and interventions to promote positive adjustment.

The collaboration addressed these questions through a series of 12 studies, including a cross-sectional survey involving 1,265 participants (Study 1) and a longitudinal study of 349 participants (Study 2). The remaining studies comprised smaller scale quantitative and qualitative designs addressing issues arising from Studies 1 and 2 in more depth [20•, 21, 22, 23].

The number of psychological factors and processes identified in the field of adjustment to disfigurement are numerous. Prior to the ARC, no consensus had been achieved between researchers in relation to which constructs and processes (and associated measures) were key 
to adjustment and assessment in clinical situations. The choice of measures in this research programme was made through achieving such a consensus between collaboration members (several of whom had previously developed their own theories and frameworks to guide research in this field), in conjunction with a thorough literature review and drawing on the clinical experience of those involved.

\section{Profiles of Adjustment}

On the basis of responses to series of psychometrically validated questionnaires completed by the 1,265 participants in Study 1, mean scores were slightly to moderately above population norms on all measures, with between 23-50\% (depending on the measure under examination) of participants scoring above norms for measures of social anxiety and distress (DAS24) [24], anxiety and depression (HADS) [25], positive and negative affect (PANAS) [26], fear of negative evaluation by others (FNE) [27] dispositional optimism/pessimism (LOT) [18], perceptions of social acceptance [20•], self-ideal discrepancies (PADQ) [28] aggression (RAQ) [29], the salience of appearance in the self concept (CARSAL) [30] and the value associated with appearance in the self concept (CARVAL) [30]. However, it was not so much the mean scores on these measures that were especially noteworthy, but rather the large range and variance in the distribution of participants' scores that were striking.

\section{Predictors of Psychological Adjustment}

A series of regression analyses identified key predictors of the variance in scores on the outcome measures used in the study (social anxiety and social avoidance assessed by scores on the DAS24; levels of anxiety and depression assessed by the HADS; positive and negative affect assessed by the PANAS). Key (and statistically significant) predictors of scores on all these outcome measures included levels of dispositional optimism (LOT), perceptions of social acceptance, fear of negative evaluation by others (FNE), the extent of the discrepancy between current perceptions of appearance and appearance ideals (PADQ) the extent to which the participants reported their disfigurement to dominate and define their sense of self (CARSAL) and the value attributed to appearance in their self concept (CARVAL).

The results of this study and the remaining studies comprising the ARC programme of research were used to inform the development of a framework to underpin the development of interventions (see [20॰] and Fig. 1 below). There remains a debate, both amongst members of the ARC and in the larger research community about the designation of 'process' and 'outcome' variables in this and other models of adjustment. Although for reasons of clarity, some of the psychological variables in the framework are conceptualised as 'process' variables and others are designated 'outcomes', this designation is inevitably somewhat contrived. The ARC are of the view that the variables designated as 'outcomes' in their framework are not simply the last links in a chain, but part of a dynamic process in which experience informs cognitions, and vice versa.

\section{Implications of the Results of the ARC Study for Face Transplantation}

The results of the ARC studies confirmed the previous research results in that the type of disfigurement and time since onset were not predictive of adjustment. The respondents in this study include an older sample than in the majority of previous research in this field (mean age 47 years, range of 18-91), offering new insights into appearance concerns in middle and older adulthood. The negative correlations between increasing age and scores for appearance distress (DAS24), aggression (RAQ), negative affect (PANAS), depression and generalized anxiety (HADS) provide a picture of positive adjustment increasing with age. Nevertheless, the variability was considerable and many older participants were concerned and distressed about their appearance. These results should not be taken as indicating that distress associated with an unusual appearance is the preserve only of the young.

Previous studies, including people with and without visible disfigurement, have in the main suggested that women are more prone to significant levels of appearance concerns and body dissatisfaction than men (see for example, [31, 32]). In the ARC study, women were more likely to have multiple areas of concern than men and levels of appearance-related anxiety. Some of these were related to the disfigurement with others relating to a variety of issues, including weight and shape. Generalized anxiety and negative affect were also higher for women. However, the effect sizes were small to medium, and men were more likely to experience feelings of aggression. Our findings stress the need for both males and females to be included in further research on disfigurement-related adjustment and distress, and in efforts to meet the needs of those adversely affected. The prevailing belief that disfigurement is less of a problem for men receives only partial support in the ARC study.

The results clearly illustrated the contribution of psychological factors and processes in adjustment and distress resulting from disfigurement and also demonstrated that the relationships are complex and dynamic. In addition to confirming the role in adjustment of socio-cognitive factors and processes identified in previous research, such as social 
Fig. 1 The ARC framework of adjustment to disfigurement

\section{ARC MODEL OF ADJUSTMENT TO VISIBLE DIFFERENCE}

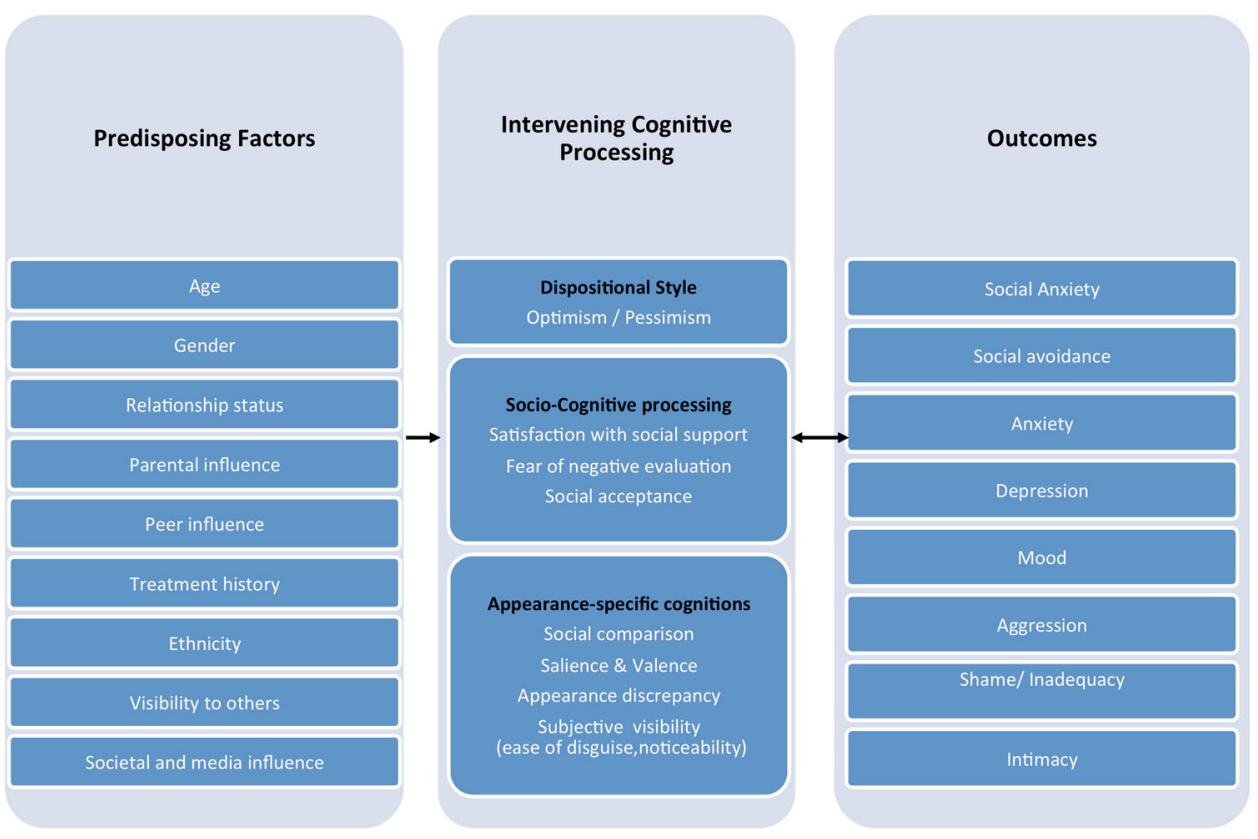

support and perceptions of social acceptance, this study has highlighted the considerable contribution of appearancespecific cognitive processes. The salience of appearance within an individual's self-concept, the value people attach to their appearance, and the gap between a person's current appearance and their ideal, accounted for a significant proportion of the variance on all outcome measures in this study, particularly in relation to levels of appearancerelated distress and dysfunction.

For all the outcome measures in the ARC study, substantial numbers of participants returned scores on standardised measures that were indicative of positive adjustment. For others, significant levels of distress were apparent. Differences in scores on the measures designated as 'outcomes' in the ARC research programme are well predicted by the variables designated as 'process' variables, and less well (or not at all) by the 'contributory factors' in the framework (see Appendix A in [20•] for more details). The framework can, therefore, be used to inform future research into individual differences in adjustment to disfigurement, and potentially, current discussions of what the psychological aims of WFT should be. A wider appreciation by WFT clinicians of the role in adjustment of the psychological factors identified in this study has the potential to help in refining the psychological goals of WFT, in choosing appropriate screening tools with which to assess prospective patients, and in the design and focus of pre- and post-procedural interventions, both in the context of psychological services for people considering and recovering from WFT, and for those assessed as being unsuitable for the procedure.

It is also important to note that the variables most strongly associated with distress in this study have been shown to be amenable to change through psychological intervention in this and related fields [20•, 33]. As an example, the variable 'dispositional optimism/pessimism' had a marked effect on levels of appearance-related anxiety and on the other outcome variables in the ARC studies. In the health psychology literature, there has been a consensus for some time that optimism is adaptive in the face of adversity, and also that it can be taught [34]. Research specifically examining optimism in the context of disfigurement has hitherto been lacking, and this study would suggest that this may be a key factor in adjustment.

The results of this research programme concur with the findings of the systematic review of the effectiveness of interventions to promote positive adjustment to disfigurement [33], which came to the tentative conclusion (in the absence of sufficient research on which to form firm conclusions) that a cognitive behavioural approach (CBT) to support and intervention holds greatest promise. This conclusion is based on the view that the approach is well suited to the cognitive issues and distress in social situations identified in the ARC program of research and in other studies. Cognitive Behavioural Therapy has also been identified as the most appropriate framework with which to conceptualise a post-WFT management plan [6]. 


\section{Conclusions}

Research since the RCS Working Party Reports published in 2004 and 2007 has confirmed that individual differences in adjustment to facial disfigurement are not well predicted by the severity or extent of the disfigurement. Neither should the age or gender of the affected person be used as a basis on which to assume levels of adjustment or distress. Instead, psychological and social factors account for the majority of the variance in adjustment. The psychological characteristics differentiating people with disfiguring conditions experiencing higher levels of distress from those who are more positively adjusted include lower levels of optimism, feelings of social acceptance and levels of satisfaction with social support, higher levels of concern about negative evaluations by others, and a view of themselves dominated by appearance-related information. These are all factors that are amenable to psychological intervention.

The guidance from the second Working Party Report included the recommendation that a thorough psychological assessment prior to transplantation, postoperatively and in the longer-term are a crucial part of patient care. This recommendation has been reinforced by clinical teams planning and undertaking WFT. While we remain in the foothills of understanding of the psychological impacts of WFT, research in the broader field of the psychology of disfigurement is of direct relevance to transplant teams as it can be used to inform the overall goals of undertaking WFT, the further development of pre- and post-procedural assessments, methods of pre-operative preparation for surgery, post-operative support, together with the development of appropriate interventions for those for whom whole face transplantation is considered inappropriate.

*Members of the ARC Nichola Rumsey (Principal Investigator), James Byron-Daniel, Rodger Charlton, Alex Clarke, Sally-Ann Clarke, Diana Harcourt, Elizabeth Jenkinson, Antje Lindenmeyer, Hayley (James) McBain, Tim Moss, Stanton Newman, Rob Newell, Krysia Saul, Emma Thomas, Andrew Thompson and Paul White.

Acknowledgments Funding for the ARC Program of Research was provided by the Healing Foundation in association with the Welton Foundation and the Worshipful Company of Tin Plate Workers. The funders were not involved in the design, data collection, analysis or interpretation of data, or the reporting of the results.

\section{Compliance with Ethics Guidelines}

Conflict of Interest Nichola Rumsey declares that she has no conflict of interest.

Human and Animal Rights and Informed Consent This article does not contain any studies with human or animal subjects performed by any of the authors.

\section{References}

Papers of particular interest, published recently, have been highlighted as:

- Of importance

1. Morris P, Bradley J, Doyal L, Earley M, Hagan P, Rumsey N. Facial transplantation: a working party report from the Royal College of Surgeons of England. Transplantation. 2004;77:330-8.

2. Morris P, Bradley A, Doyal L, Earley M, Hagen P, Milling M, Rumsey N. Face transplantation: a review of the technical, immunological, psychological and clinical issues with recommendations for good practice. Transplantation. 2007;83(2):109-28.

3. Coffman KL, Siemionow MD. Face transplantation: psychological outcomes at three year follow-up. Psychosomatics. 2013;54:372-8.

4. Chang G, Pomahac B. Psychosocial changes 6 months after face transplantation. Psychosomatics. 2013;54(4):367-71.

5. Coffman K, Gordon C, Siemionow M. Psychological outcomes with face transplantation: overview and case report. Curr Opin Organ Transplant. 2010;15(2):236-40.

6. Brill S, Clarke A, Veale D, Butler P. Psychological management and body image issues in facial transplantation. Body Image. 2006;3(1):1-15.

7. Rumsey N, Harcourt D. Body image and disfigurement: issues and intervention. Body Image. 2004;1:83-7.

8. Stilley C, Flynn W, Sereika S, et al. Pathways of psychosocial factors, stress and health outcomes after liver transplantation. Clin Transplant. 2012;26(2):216-22.

9. Mohammad S, Hormaza L, Neighbors K, et al. Health status in young adults two decades after pediatric liver transplantation. Am J Transplant. 2012;12(6):1486-95.

10. Baranyi A, Krauseneck T, Rothenhaeusler H. Overall mental distress and health-related quality of life after solid-organ transplantation: results from a retrospective follow-up study. Health Qual Life Outcomes. 2013;11:235-9.

11. Shanmugarajah K, Hettiaratchy S, Clarke A, Butler P. Clinical outcomes of facial transplantation: a review. Int $\mathrm{J}$ Surg. 2011;9:600-7.

12. - Rumsey N, Harcourt D. The Oxford handbook of appearance. Oxford: Oxford University Press; 2012. Edited handbook summarising current knowledge about psychological adjustment to disfigurement. Includes 50 chapters organised into sections on 'who is affected by appearance concerns, in what way, and why', 'individual differences in adjustment and distress', 'experiences of people who have a visible difference', 'interventions at an individual or group level', and more.

13. Ong J, Clarke A, Johnson M, White P, Withey S, Butler P. Does severity predict distress: the relationship between subjective and objective measures of severity in patients treated for facial lipoatrophy. Body Image: Int J Res. 2007;4:239-48.

14. White CA. Body image issues in oncology. In: Cash TF, Pruzinsky T, editors. Body image: a handbook of theory, research and clinical practice. London: Guilford Press; 2002.

15. Thompson A, Kent G. Adjusting to disfigurement: processes involved in dealing with being visibly different. Clin Psychol Rev. 2001;21:663-83.

16. Goffman E. Stigma: notes on the management of spoilt identity. Englewood Cliffs: Prentice Hall; 1963.

17. Kent G, Thompson A. The development and maintenance of shame in disfigurement: implications for treatment. In: Gilbert P, Miles J, editors. Body shame: conceptualisation, research and treatment. Hove: Brunner-Routledge; 2002. p. 106-16. 
18. Leary MR. A brief version of the fear of negative evaluation scale. Pers Soc Psychol Bull. 1983;9:371-5.

19. Newell RJ. Body image and disfigurement care. London: Routledge; 2000.

20. - Clarke A, Thompson A, Jenkinson E, Rumsey N, Newell R CBT for appearance anxiety: psychosocial interventions for anxiety due to visible difference. Chichester: WileyBlackwell; 2013. Evidence based handbook to guide interventions describing CBT techniques designed to alleviate distress in those with disfigurement. Also includes the evidence base for the ARC research programme (see Appendix A).

21. Egan K, Harcourt D, Rumsey N, The Appearance Collaboration. A qualitative study of the experiences of people who identify themselves as having adjusted positively to a visible difference. J Health Psychol. 2011;16:739-49.

22. Thompson A, Clarke A, Newell R, Gawkrodger D, The Appearance Collaboration. Vitiligo linked to stigmatization in British South Asian women: a qualitative study of the experiences of living with vitiligo. Br J Dermatol. 2010;163:481-6.

23. James H, Jenkinson E, Harrad R, Ezra D, Newman S, The Appearance Collaboration. Appearance concerns in ophthalmic patients. Eye. 2011;25:1039-44.

24. Carr T, Moss T, Harris D. The DAS24: a short form of the Derriford Appearance Scale DAS59 to measure individual responses to living with problems of appearance. $\mathrm{Br} \mathrm{J}$ Health Psychol. 2005;10:285-98.

25. Zigmond AS, Snaith RP. The hospital anxiety and depression scale. Acta Psychiatr Scand. 1983;67:361-70.
26. Watson D, Clark LA, Tellagan A. Development and validation of brief measures of positive and negative affect: the PANAS scales. J Pers Soc Psychol. 1988;47:1063-70.

27. Scheier MF, Carver CS, Bridges MW. Distinguishing optimism from neuroticism (and trait anxiety, self-mastery, and selfesteem): a re-evaluation of the life orientation test. J Pers Soc Psychol. 1994;67:1063-78.

28. Altabe M, Thompson J. Body image: a cognitive self-schema. Cognit Ther Res. 1996;20:171-93.

29. Bryant FB, Smith BD. Refining the architecture of aggression: a measurement model for the buss-perry aggression questionnaire. J Res Pers. 2001;35:138-67.

30. Moss T, Rosser B. The moderated relationship of appearance valence on appearance self consciousness: development ad testing of new measures of appearance schema component. PLos One. 2012;7(11):e50605.

31. Cash TF, Melnyk SE, Hrabosky JI. The assessment of body image investment: an extensive revision of the body schemas inventory. Int J Eat Disord. 2004;25:305-16.

32. Harris D, Carr A. Prevalence of concern about physical appearance in the general population. Br J Plast Surg. 2001;54:223-6.

33. Bessell A, Moss T. Evaluating the effectiveness of psychosocial interventions for individuals with visible differences: a systematic review of the empirical literature. Body Image: Int $\mathrm{J}$ Res. 2007;4(2):227-38.

34. Reich J, Zautra A, Hall J, editors. Handbook of adult resilience. New York: Guilford Press; 2010. 